\title{
Health, Functional and Nutritional Determinants of Falls Experienced in the Previous Year-A Cross-Sectional Study in a Geriatric Ward
}

\author{
Lukasz Magnuszewski $^{1,2}$, Marta Swietek ${ }^{1,2}$, Agnieszka Kasiukiewicz ${ }^{2,3}$ (D), \\ Bartlomiej Kuprjanowicz ${ }^{2}$, Jan Baczek ${ }^{1}$ and Zyta Beata Wojszel ${ }^{2,3, * \text { (D) }}$ \\ 1 Doctoral Studies, Department of Geriatrics, Faculty of Health Sciences, Medical University of Bialystok, \\ 15-471 Białystok, Poland; magnuszewskilukas@gmail.com (L.M.); marta.swietek@umb.edu.pl (M.S.); \\ janbaczek@gmail.com (J.B.) \\ 2 Department of Geriatrics, Hospital of the Ministry of Interior and Administration in Bialystok, \\ 15-471 Bialystok, Poland; akasiukiewicz@o2.pl (A.K.); barti.bial@wp.pl (B.K.) \\ 3 Department of Geriatrics, Medical University of Bialystok, 15-471 Bialystok, Poland \\ * Correspondence: wojszel@umb.edu.pl; Tel.: +48-85-869-4982
}

Received: 15 June 2020; Accepted: 30 June 2020; Published: 2 July 2020

\begin{abstract}
Falls are a serious health problem in older adults. A limited number of studies assessed their multifactorial nature in geriatric ward patients. The aim of this study is to explore health, functional and nutritional correlates of experiencing fall(s) in the previous year by older inpatients. A cross-sectional study of patients admitted to the department of geriatrics was conducted. A "faller status" was defined based on positive history of falls in the previous 12 months. Health, functional and nutritional factors associated with falls were evaluated, and multivariable logistic regression analysis models were built. A total of 358 patients (median age 82 (IQR 76-86) years, 77.9\% women) were recruited, $43.9 \%$ of whom reported falls. The "fallers" presented with a significantly higher number of chronic diseases, higher prevalence of Parkinson's disease, peripheral arterial disease, chronic osteoarthritis, more frequently reported urinary incontinence and were dependent on others for daily living activities. They had significantly worse results for the assessment of gait, balance and frailty status. The Mini Nutritional Assessment-Short Form scores and the mean value of serum albumin were significantly lower in the fallers' group. Parkinson's disease $(\mathrm{OR}=2.82, \mathrm{CI}-1.07-7.45$; $p=0.04)$ and osteoarthritis ( $\mathrm{OR}=2.08, \mathrm{CI}-1.02-4.23 ; p=0.04$ ) were the main variables for the outcome prediction, according to the direct multivariable logistic regression analysis. Our findings suggest that Parkinson's disease and osteoarthritis are the main predictors independently associated with a history of falls in patients admitted to the geriatric ward, although the influence of some factors may be underestimated due to the tendency of not taking the history of falls in very frail, functionally dependent and bedridden individuals.
\end{abstract}

Keywords: fall risk factors; fall detection; fall epidemiology; geriatrics; older patients

\section{Introduction}

Falls are a major health problem in older adults. A high percentage of the geriatric population experienced a fall each year [1,2]. Falls are connected with high morbidity, increased odds for hospitalization caused by serious injuries, increased risk of mortality, fear of falling (post-fall syndrome), and lead to lower quality of life [3].

Many intrinsic and extrinsic factors can contribute to or cause falls. The intrinsic factors include gait and balance disorders, cognitive deterioration, abnormal nutritional status, orthostatic hypotension and different medical conditions such as Parkinson's disease, stroke and its consequences 
or chronic osteoarthritis, among others [4-9]. The extrinsic factors include maladjustment of the living environment to the disability of an older person, polypharmacy, taking certain groups of a drug for treatments (such as psychotropics, diuretics or antihypertensive medications) [10-12]. Furthermore, sarcopenia-defined as low skeletal muscle mass, strength, and physical performance has been pointed recently as a potentially modifiable risk factor of falls in older people [13]. Both groups of factors-internal and external-are interrelated.

The aim of the study was to assess clinical health, functional and nutritional characteristics associated with the history of pre-hospitalization falls in geriatric inpatients, taking into account the multifactorial nature of this "geriatric syndrome" [14]. To the authors' knowledge, a limited number of studies examined determinants of faller status in patients hospitalized in a geriatric ward from the multifactorial perspective, based on the comprehensive geriatric assessment [15]. The problem of falls was most often assessed in community-dwelling older people and the risk factors taken into account were relatively few [2]. Studies carried out in hospital departments concentrated, for instance, on the risk of falls in patients who have fallen in hospital, in the context of the possibility of preventing falls in inpatients $[16,17]$, selectively analyzed some risk factors for falls, such as the type of medication taken [18] or assessed the impact of the risk of falls on prognosis after discharge from hospital [19].

\section{Materials and Methods}

Our study is a secondary analysis of the data collected across the cross-sectional study on frailty and multimorbidity in patients of the department of geriatrics (Hospital of the Ministry of Interior and Administration in Bialystok, Poland) [20]. All consecutive patients, admitted for the first time to the department of geriatrics on the turn of 2014 and 2015, took part in it. The department of geriatrics is a subacute care ward; patients are admitted primarily electively. Most often, they are burdened with chronic diseases and various disabilities. One of the main goals of their stay in the ward is to conduct a comprehensive geriatric assessment and to create a long-term care plan. For this reason, in most cases, it is not possible to indicate one specific reason for hospitalization. Patient examinations were carried out by permanent employees of the ward: geriatricians and specialists in internal medicine, nurses specialized in geriatrics or long-term care, a clinical neuropsychologist and a physiotherapist.

The analysis included patients who-alone or represented by a guardian-answered the question about the occurrence of falls in the last 12 months. Participants who confirmed such incidents were treated as having a "faller" status, and those who denied them as "non-fallers".

\subsection{Patient Characteristics}

Sociodemographic characteristics included age, gender, place of residence (urban/rural). History taking included information on 15 chronic diseases (peripheral arterial disease, ischemic heart disease, chronic cardiac failure, myocardial infarction, hypertension, atrial fibrillation, history of transient ischemic attack (TIA) or stroke, chronic obstructive pulmonary disease, diabetes or prediabetes, neoplasm, dementia, parkinsonism, chronic osteoarthritis, osteoporosis and chronic renal disease), drug for treatments taken before hospitalization that could influence the risk of falls (antihypertensive drugs: angiotensin converting enzyme inhibitors (ACE-Is)/ angiotensin II receptor blockers (ARBs), beta-blockers, calcium channel blockers, diuretics, $\alpha 1$-blockers; digoxin; lipid lowering drugs; antidepressants: selective serotonin reuptake inhibitors (SSRI); neuroleptics: in general and quetiapine separately; pro-cognitive medications: memantine, acetylcholinesterase inhibitors (AChE-Is), benzodiazepines (BDA); non-steroidal anti-inflammatory drugs (NSAIDs)) and hospitalizations in the last 12 months. Information obtained from the patient was verified by an interview with his or her guardian, by a thorough clinical examination, and by a review of all of the patient's medical records available. 


\subsection{Measurements}

The functional profile of a patient was created based on the results of comprehensive geriatric assessment routinely conducted at the department. Risk of recurrent falls was assessed with previously validated tests-the Performance-Oriented Mobility Assessment (POMA) [21] and the Timed Up-and-Go test (TUG) [22]. Walking speed was measured during the $4.6 \mathrm{~m}$ walk from standing position in usual gait speed (the fastest time of 2 trials was used). Hand grip strength was measured in the dominant hand according to the Southampton protocol, using a hand-held hydraulic dynamometer DHD-1 (SAEHAN, Changwon, Korea) [23]. The ability to perform basic activities of daily living was assessed with the Barthel index [24] and instrumental activities of daily living (IADL) with 6 items of the Duke Older American Resources and Services (OARS) I-ADL [25]. Cognitive abilities were evaluated using the Abbreviated Mental Test Score (AMTS) [26] and the possibility of depression with the 15-item Geriatric Depression Scale (GDS) [27]. The diagnosis of dementia at discharge was made on the basis of the neuropsychologist's examination during the patient's hospital stay. The 7-item Canadian Study of Health and Aging Clinical Frailty Scale (CSHA-CFS) was used to asses frailty [28]. Risk of malnutrition was determined with the Mini Nutritional Assessment Short form (MNA-SF) [29]. Body mass ndex (BMI) was measured according to the standard procedure.

Systolic and diastolic blood pressure was measured at admission. Orthostatic hypotension occurrence was assessed with active standing test performed by a physiotherapist in the morning on the second day of the patient's hospital stay. After a resting period of $10 \mathrm{~min}$, blood pressure was measured electronically (Automatic Philips IntelliVue MP5 Monitor, Amsterdam, The Netherlands) on the non-dominant arm with the patient lying down. The patient was then asked to stand up, and the blood pressure was measured again during the first and the third minute after standing [30]. If the patient was not able to stand, the measurement was made in a sitting position.

Data on vitamin B12, serum sodium, creatinine, albumin, and hemoglobin levels was extracted from patients' medical records. Renal function was assessed with glomerular filtration rate-GFR, counted using the Chronic Kidney Disease Epidemiology Collaboration (CKD-EPI) formula [31].

\subsection{Study Parameters}

Multimorbidity was defined as the co-occurrence of 5 or more diseases of the 15 listed above. Polypharmacy was defined as 5 or more drugs taken. Severe frailty was defined as a CFS score of 6 or 7 [28]. Orthostatic hypotension was defined by a drop in blood pressure of at least $20 \mathrm{mmHg}$ for systolic blood pressure and at least $10 \mathrm{mmHg}$ for diastolic blood pressure within 3 min of standing up. Chronic kidney disease, i.e., stages 3, 4 and 5 CKD according to Kidney Disease Outcome Quality Initiative (KDOQI), was diagnosed if the GFR was $<60 \mathrm{~mL} / \mathrm{min} / 1.73 \mathrm{~m}^{2}$. Anemia was diagnosed if the hemoglobin level was below $8.7-\mathrm{mmol} / \mathrm{L}$ in men and below $7.5-\mathrm{mmol} / \mathrm{L}$ in women. Patients with $\mathrm{BMI} \geq 30 \mathrm{~kg} / \mathrm{m}^{2}$ were classified as obese, and with $\mathrm{BMI}<24 \mathrm{~kg} / \mathrm{m}^{2}$ as being at risk for malnutrition. Malnutrition was also suspected if the MNA-SF score was below 8. Dynapenia (or probable sarcopenia) was derived from the handgrip strength and diagnosed in men if grip strength was lower than $27 \mathrm{~kg}$ and in women if it was lower than $16 \mathrm{~kg}$ [32]. It was classified as severe based on physical performance; if low grip strength was accompanied by gait speed equal or lower than $0.8 \mathrm{~m} / \mathrm{s}$, and/or TUG equal or higher than $20 \mathrm{~s}$, the patient was diagnosed as severely dynapenic.

\subsection{Statistical Analysis}

The IBM SPSS Version 18 Software suit (SPSS, Chicago, IL, USA) and STATISTICA 13.3 software package (TIBCO Software, Palo Alto, CA, USA) were used to analyze the data collected. The Shapiro-Wilk test was used to assess the distribution of variables. Data were presented as means (M) and standard deviation (SD) for normally distributed continuous variables, as medians (Me) and interquartile range (IQR) for not normally distributed ones and the number of cases and percentage for categorical variables. Proportions were compared using $\chi^{2}$ tests or Fisher's exact test, as appropriate, 
while the independent samples Student's $t$-test and the Mann-Whitney U test were used to compare the distribution of continuous variables. It was followed by a multivariable logistic regression including all predictors with a $p$-value less than 0.2 and without significant multicolinearity effect. The variance inflation factor was used to identify correlation between independent variables and the strength of that correlation. Missing values were omitted and statistics in such cases were calculated for the adequately reduced groups. A $p$-value of less than 0.05 was regarded as significant.

\subsection{Ethics Approval}

The source study was approved by the Ethics Committee at the Medical University of Bialystok (no R-I-002/305/2013). All procedures performed in the study were in accordance with the ethical standards of the Medical University of Bialystok research committee and with the Helsinki declaration and its later amendments. The study can be classified as a study of 'usual practice'. All study participants gave their informed consent to participate in it.

\section{Results}

\subsection{Study Cohort Characteristics}

A total of $358(86.1 \%)$ patients of 416 hospitalized during the study period-or their guardians-answered the question about the occurrence of falls in the last 12 months and were included in the analysis (Figure 1). Falls in the previous year were reported by $43.9 \%$ of them. The median age of patients was 82 years (IQR 77-86) and most of them were above 75 years of age $(83.2 \%)$ and female $(77.9 \%)$.

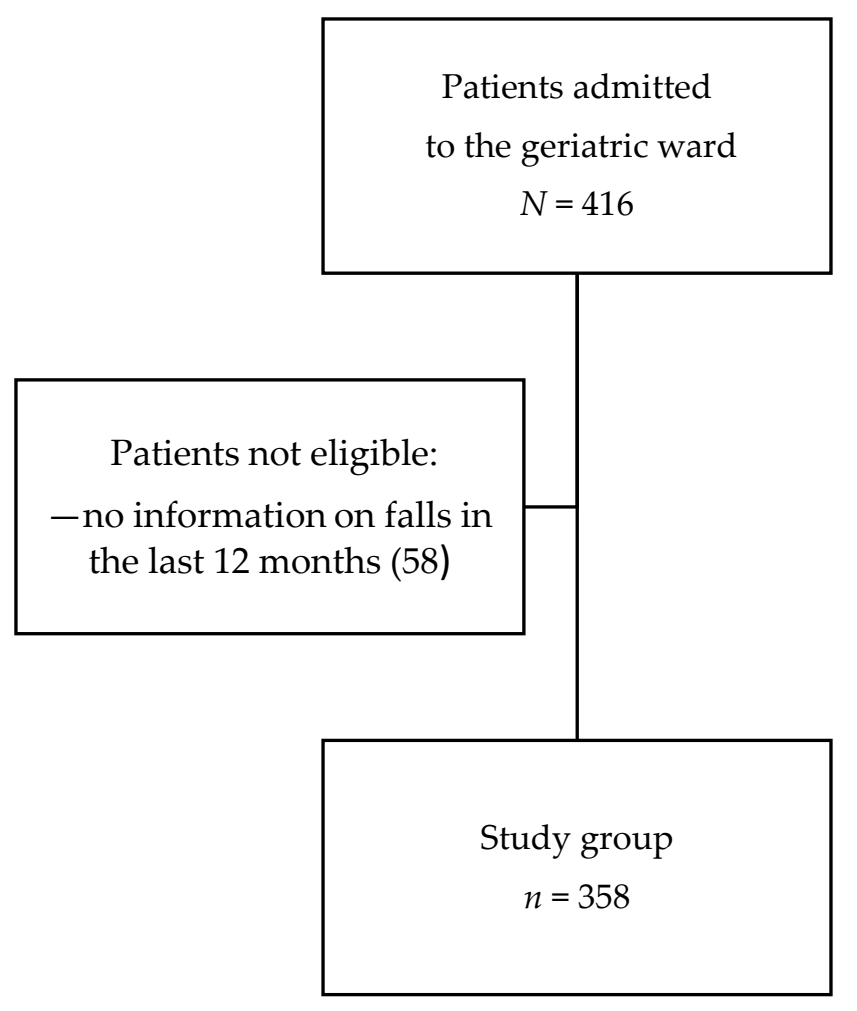

Figure 1. Flow chart of patient enrollment.

The characteristics of the study groups-fallers and non-fallers-are presented in Table 1 (sociodemographic and health characteristics) and Table 2 (nutritional status and functional characteristics). They did not differ in age, gender and place of residence. The groups differed significantly in the number of chronic diseases (fallers: Me 5.0, IQR 3.0-6.0 versus non-fallers: Me 
4.0, IQR 3.0-6.0, $p=0.02$ ), but not in the percentage of multimorbidity. The percentage of some diseases was significantly higher in the fallers group: Parkinson's disease (fallers: $17.8 \%$ versus $6.5 \%$ in non-fallers, $p<0.001$ ), peripheral arterial disease (fallers: $26.8 \%$ versus $16.4 \%$ in non-fallers, $p=0.02$ ) and chronic osteoarthritis (fallers: $82.8 \%$ versus $73.6 \%$ in non-fallers, $p=0.04$ ), but the percentage of dementia, hypertension, ischemic heart disease, history of myocardial infarction, congestive heart failure, TIA/ stroke, diabetes, osteoporosis and chronic kidney disease was not substantially different in both groups. The median number of medications taken, and the percentage of polypharmacy also did not significantly differ. We did not notice any dissimilarities in the frequency of taking any type of medication. Only in the case of quetiapine, the difference was on the verge of statistical significance (fallers: $14.5 \%$ versus $8.1 \%$ in non-fallers, $p=0.06$ ). Median systolic and diastolic blood pressure value at admittance and percentage of orthostatic hypotension were similar in fallers and non-fallers. The groups did not differ in median levels of vitamin B12, serum sodium, serum creatinine, hemoglobin and in mean value of GFR. The percentage of anemia patients was also not significantly different in both groups.

Table 1. Characteristics of study participants—sociodemographic and health correlates of falls.

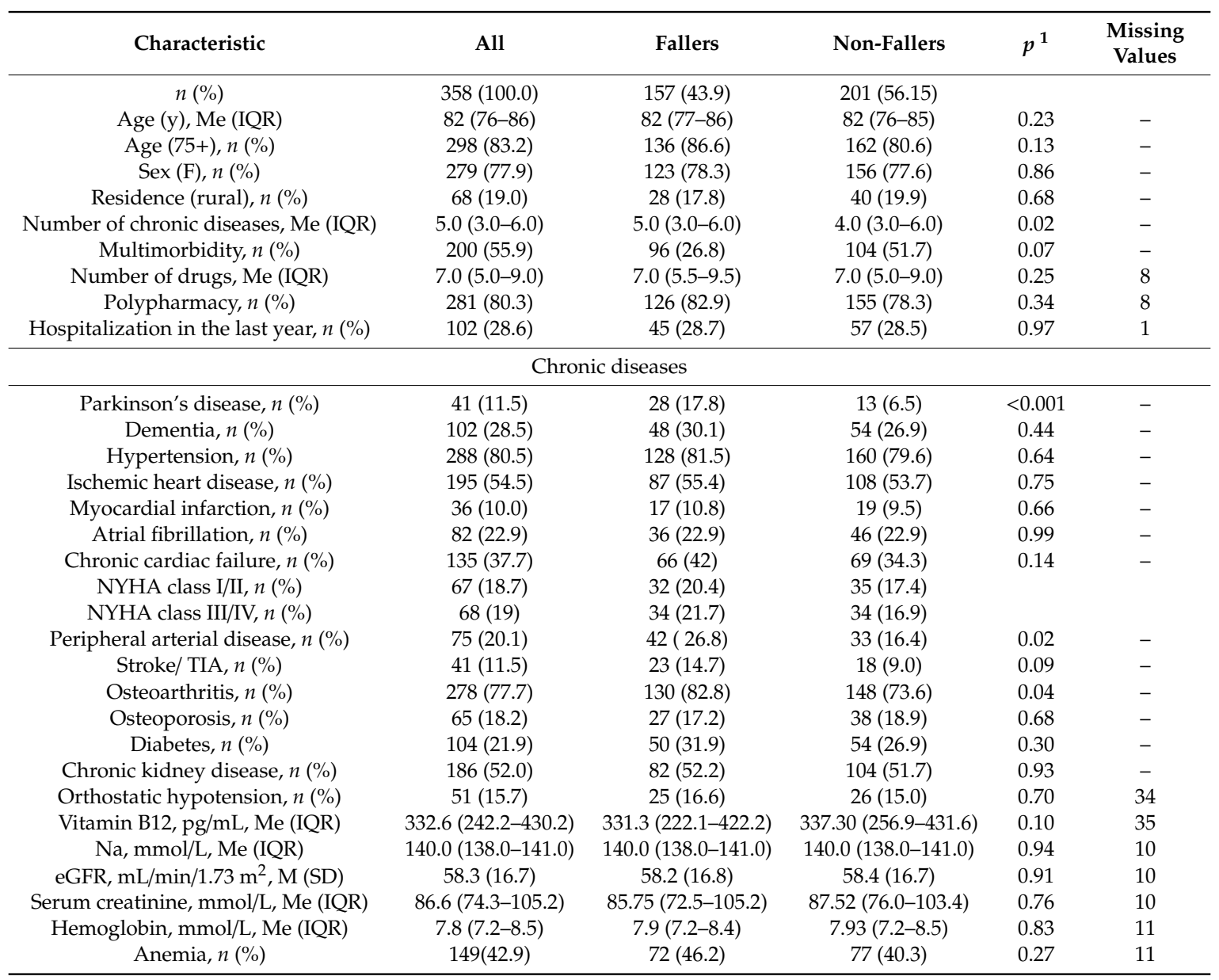


Table 1. Cont.

\begin{tabular}{|c|c|c|c|c|c|}
\hline Characteristic & All & Fallers & Non-Fallers & $p^{1}$ & $\begin{array}{c}\text { Missing } \\
\text { Values }\end{array}$ \\
\hline \multicolumn{6}{|c|}{ Medications } \\
\hline Thyroid hormones, $n(\%)$ & $26(7.5)$ & $11(7.2)$ & $15(7.6)$ & 0.89 & 9 \\
\hline Antithyroid drugs, $n(\%)$ & $2(06)$ & - & $2(1.0)$ & 0.51 & 9 \\
\hline Quetiapine, $n(\%)$ & $38(10.9)$ & $22(14.5)$ & $16(8.1)$ & 0.06 & 9 \\
\hline ACE-Is/ ARBs, $n(\%)$ & $231(66.2)$ & $95(62.5)$ & $136(69.0)$ & 0.21 & 9 \\
\hline Memantine, $n(\%)$ & $11(3.2)$ & $3(2.0)$ & $8(4.0)$ & 0.27 & 9 \\
\hline AChE-Is, $n(\%)$ & $35(10.0)$ & $17(11.2)$ & $18(9.1)$ & 0.53 & 9 \\
\hline B-blockers, $n(\%)$ & $222(63.6)$ & $98(64.5)$ & $124(63.0)$ & 0.77 & 9 \\
\hline Calcium channel blockers, $n(\%)$ & $102(29.2)$ & $46(30.3)$ & $56(28.4)$ & 0.70 & 9 \\
\hline SSRI, $n(\%)$ & $98(28.0)$ & $49(32.2)$ & $49(24.9)$ & 0.13 & 9 \\
\hline NSAIDs, $n(\%)$ & $24(6.9)$ & $12(7.9)$ & $12(6.1)$ & 0.53 & 9 \\
\hline
\end{tabular}

${ }^{1}-\chi^{2}$ test or Fisher's exact test, as appropriate, for categorical variables; $t$-test for independent samples or Mann-Whitney test for continuous or interval variables. In all analyses a two-tailed $P$-value of less than 0.05 was regarded as significant. ACE-Is-angiotensin converting enzyme inhibitors; AChE-I-acetylcholinesterase inhibitors; ARBs-angiotensin II receptor blockers; BDA—-benzodiazepines; BP-blood pressure-eGFR-glomerular filtration rate; IQR —interquartile range; $\mathrm{M}$-mean value; Me-median value; $n$-number of cases; Na—serum sodium; NSAIDs—non-steroidal anti-inflammatory drugs; SSRI—selective serotonin reuptake inhibitors; SD—standard deviation; TIA — transient ischemic attack.

Table 2. Characteristics of study participants-functional and nutritional correlates of falls.

\begin{tabular}{|c|c|c|c|c|c|}
\hline Characteristic & All & Fallers & Non-Fallers & $p^{1}$ & Missing Values \\
\hline$n(\%)$ & $358(100.0)$ & $157(43.9)$ & $201(56.15)$ & & \\
\hline Barthel index, Me (IQR) & $95(80-100)$ & $90(75-95)$ & $95(85-100)$ & 0.004 & 6 \\
\hline IADL, Me (IQR) & $8.0(5.0-11.0)$ & $7.0(4.0-10.5)$ & $9.0(6.0-12.0)$ & 0.01 & 10 \\
\hline AMTS, Me (IQR) & $8.0(6.0-9.0)$ & $8.0(7.0-9.0)$ & $8.0(6.0-9.0)$ & 0.58 & 21 \\
\hline GDS, Me (IQR) & $6.0(3.0-10.0)$ & $7.0(3.0-10.0)$ & $6.0(3.0-9.0)$ & 0.34 & 34 \\
\hline Handgrip, kg, Me (IQR) & $18.4(14.1-23.0)$ & $18.1(13.9-22.3)$ & $18.8(14.3-24.3)$ & 0.22 & 32 \\
\hline men, kg, Me (IQR) & $26(21.0-32.3)$ & $26.1(21.5-29.6)$ & $30.0(23.0-35.5)$ & 0.047 & 12 \\
\hline women, kg, Me (IQR) & $16.7(12.9-20.5)$ & $16.8(12.7-19.9)$ & $17.3(13.5-21.3)$ & 0.2 & 20 \\
\hline Dynapenia, $n(\%)$ & $143(43.9)$ & $73(47.4)$ & $70(40.7)$ & 0.26 & 32 \\
\hline Severe dynapenia, $n(\%)$ & $95(32.8)$ & $48(36.4)$ & $47(29.7)$ & 0.26 & 68 \\
\hline Gait speed, m/s, Me (IQR) & $0.65(0.40-0.95)$ & $0.60(0.37-0.86)$ & $0.71(0.43-1.05)$ & 0.01 & 49 \\
\hline Gait speed $\leq 0.8 \mathrm{~m} / \mathrm{s}, n(\%)$ & $200(64.7)$ & $103(71.0)$ & $97(59.1)$ & 0.03 & 49 \\
\hline POMA, Me (IQR) & $23.0(18.0-28.0)$ & $22.0(17.0-28.0)$ & $25.0(19.0-28.0)$ & 0.005 & 49 \\
\hline TUG, s, Me (IQR) & $17.1(11.7-27.2)$ & $17.4(13.0-28.3)$ & $16.5(11.3-25.2)$ & 0.25 & 64 \\
\hline TUG $\geq 20 \mathrm{~s}, n(\%)$ & $121(41.2)$ & $55(41.7)$ & $66(40.7)$ & 0.91 & 64 \\
\hline CFS, Me (IQR) & $4.0(4.0-5.0)$ & $5.0(4.0-5.0)$ & $4.0(4.0-5.0)$ & 0.03 & - \\
\hline Severe frailty, $n(\%)$ & $58(16.2)$ & $31(19.7)$ & $27(13.4)$ & 0.11 & - \\
\hline Urinary incontinence, $n(\%)$ & $146(41.4)$ & $78(49.7)$ & $68(34.7)$ & 0.004 & 5 \\
\hline BMI, kg/m2, M (SD) & $29.35(5.99)$ & $29.33(5.97)$ & $29.37(6.02)$ & 0.92 & 34 \\
\hline $\mathrm{BMI}<24 \mathrm{~kg} / \mathrm{m}^{2}, n(\%)$ & $59(18.2)$ & $26(18.7)$ & $33(17.9)$ & 0.84 & 34 \\
\hline $\mathrm{BMI}>30 \mathrm{~kg} / \mathrm{m}^{2}, n(\%)$ & $136(42.0)$ & $57(41.0)$ & $79(42.7)$ & 0.76 & 34 \\
\hline $\mathrm{MAC}, \mathrm{cm}, \mathrm{M}(\mathrm{SD})$ & $28.2(4.0)$ & $28.1(4.0)$ & $28.3(3.9)$ & 0.82 & 47 \\
\hline $\mathrm{MAC} \leq 22 \mathrm{~cm}, n(\%)$ & $73(23.5)$ & $35(23.8)$ & $38(23.2)$ & 0.89 & 47 \\
\hline $\mathrm{CC}, \mathrm{cm}, \mathrm{M}(\mathrm{SD})$ & $34.8(4.5)$ & $34.6(4.7)$ & $35.0(4.4)$ & 0.50 & 47 \\
\hline $\mathrm{CC}<31 \mathrm{~cm}, n(\%)$ & $53(17.0)$ & $29(19.7)$ & $24(14.6)$ & 0.23 & 47 \\
\hline Albumin, g/L, M (SD) & $39.3(3.7)$ & $39.0(4.0)$ & $39.5(4.0)$ & 0.03 & 24 \\
\hline MNA-SF, Me (IQR) & $12.0(10.0-13.0)$ & $12.0(9.0-13.0)$ & $13.0(10.0-14.0)$ & 0.04 & 10 \\
\hline MNA-SF score $<8, n(\%)$ & $46(13.2)$ & $21(13.6)$ & $25(12.9)$ & 0.84 & 10 \\
\hline
\end{tabular}

$1-\chi^{2}$ test or Fisher's exact test, as appropriate, for categorical variables; $t$-test for independent samples or Mann-Whitney test for continuous or interval variables. In all analyses a two-tailed $P$-value of less than 0.05 was regarded as significant. AMTS-Abbreviated Mental Test Score; BMI-body mass index; CC—calf circumference; CFS-7-point Clinical Frailty Scale; IADL_instrumental activities of daily living; IQR-interquartile range; $\mathrm{M}$-mean value; Me-median value; MAC — mid arm circumference; MNA-SF- Mini Nutritional Assessment Short Form; $n$-number of cases; POMA—Performance Oriented Mobility Assessment; TUG-Timed Up-and-Go test; SD—standard deviation. 
Similar percentages of fallers and non-fallers were hospitalized in the last year (information on the average number of hospitalizations was not available). The Barthel index, the Duke OARS and the MNA-SF scales scores were significantly lower in the fallers' group, whereas the AMTS and GDS scores were not materially different. Urinary incontinence was reported significantly more frequently in the fallers' group. The gait speed, the POMA, and the TUG results were significantly worse in the fallers' group and the percentage of patients with gait speed $\leq 0.8 \mathrm{~m} / \mathrm{s}$ was significantly higher $(71.0 \%$ versus $59.1 \%$ in non-fallers, $p=0.03$ ). The handgrip strength did not differ significantly between fallers and non-fallers, although such a difference was observed in men. Despite that, no difference was observed in the percentage of patients with dynapenia, as well as those classified as severely dynapenic between fallers and non-fallers. The medium level of frailty status classified with the CFS was significantly higher in fallers (5.0, IQR 4.0-5.0 vs. 4.0, IQR 4.0-5.0 in non-fallers, $p=0.03$ ), but the groups did not differ significantly in the percentage of patients assessed as severely frail. The mean BMI as well as percentages of patients with BMI below $24.0 \mathrm{~kg} / \mathrm{m}^{2}$ and with BMI above $30 \mathrm{~kg} / \mathrm{m}^{2}$ were not significantly different in fallers and non-fallers. The mean value of serum albumin was notably lower in fallers: 39.0 $\pm 4.0-\mathrm{g} / \mathrm{L}$ vs. $39.5 \pm 4.0 \mathrm{~g} / \mathrm{L}$ in non-fallers, $p=0.03$, but the groups did not differ significantly in the percentages of patients at risk of malnutrition according to the MNA-SF score.

\subsection{Independent Predictors of Being a Faller}

A direct multivariable logistic regression analysis was carried out on faller status as outcome and 21 predictors: age 75+, multimorbidity, cardiac heart failure, peripheral arterial disease, history of stroke/TIA, Parkinson's disease, chronic osteoarthritis, POMA, Barthel Index, IADL score, gait speed, CFS status, urinary incontinence, MNA-SF score, albumin value, vitamin B12 level and taking certain medications (quetiapine, vitamin D, diuretics, BDA and SSRI) (Table 3). A few variables meeting the criterion $p<0.2$ (number of chronic diseases, gait speed $\leq 0.8 \mathrm{~m} / \mathrm{s}$ and severe frailty) were not included in the logistic regression after testing for correlation with other variables and for their multicollinearity effect. The handgrip variable was introduced into the regression model, although it did not meet criteria ( $p=0.22$ ) because when it was separated by sex, it did it. Significantly higher odds for faller status was observed only for Parkinson's disease (odds ratio, 2.82;95\% CI, 1.07-7.45; $p=0.04$ ) and osteoarthritis (odds ratio, 2.08; 95\% CI, 1.02-4.23; $p=0.04$ ).

A test of this model against a constant-only model was statistically reliable, $\chi(21, N=265)=$ $32.90, p<0.047$, indicating that the characteristics, as a set, reliably distinguished between fallers and non-fallers. An overall prediction success rate in logistic regression constituted $64.2 \%$, with $60.2 \%$ of fallers and $67.9 \%$ of non-fallers predicted correctly.

\subsection{Study Participants and Nonparticipants}

Patients hospitalized in the geriatric ward during the study period, but excluded from the analysis because of the missing data on falls in the previous 12 months did not differ from study participants in age, gender and in a number of characteristics, but were more often severely frail, demented, and had significantly worse nutritional and functional parameters (Table 4). 
Table 3. Results of the direct logistic regression model indicating characteristics associated with falls.

\begin{tabular}{ccc}
\hline Predictors & OR (95\% CI) & $p$ \\
\hline Age, 75+ years & $1.17(0.54-2.53)$ & 0.69 \\
Multimorbidity & $1.01(0.52-1.98)$ & 0.97 \\
Chronic cardiac failure & $1.67(0.88-3.14)$ & 0.12 \\
Peripheral arterial disease & $0.90(0.41-2.00)$ & 0.80 \\
Stroke/TIA & $1.82(0.71-4.67)$ & 0.22 \\
Parkinson's disease & $2.82(1.07-7.45)$ & $\mathbf{0 . 0 4}$ \\
Osteoarthritis & $2.08(1.02-4.23)$ & $\mathbf{0 . 0 4}$ \\
POMA & $0.95(0.87-1.02)$ & 0.16 \\
Barthel index & $1.01(0.98-1.03)$ & 0.53 \\
IADL & $0.99(0.89-1.12)$ & 0.96 \\
Gait speed & $0.78(0.18-3.39)$ & 0.69 \\
CFS & $0.76(0.50-1.16)$ & 0.21 \\
Urinary incontinence & $1.37(0.75-2.49)$ & 0.31 \\
MNA-SF & $1.02(0.91-1.15)$ & 0.73 \\
Albumin & $1.00(0.92-1.09)$ & 0.96 \\
Vitamin B12 & $0.99(0.99-1.00)$ & 0.12 \\
Quetiapine & $1.66(0.64-4.31)$ & 0.30 \\
Vitamin D & $1.08(0.54-2.17)$ & 0.82 \\
Diuretics & $0.69(0.39-1.21)$ & 0.19 \\
BDA & $1.29(0.54-3.08)$ & 0.56 \\
SSRI & $1.30(0.69-2.46)$ & 0.42 \\
\hline
\end{tabular}

Bold values indicate statistical significance at the $p<0.05$ level. BDA-benzodiazepines; CFS-Clinical Frailty Scale; CI-confidence interval; IADL-instrumental activities of daily living; MNA-SF-Mini Nutritional Assessment Short Form; OR—odds ratio; POMA—Performance Oriented Mobility Assessment-SSRI—selective serotonin reuptake inhibitor; TIA—transient ischemic attack.

Table 4. Characteristics of participants and nonparticipants of the study.

\begin{tabular}{cccc}
\hline Parameter & Participants & Nonparticipants & $p^{\mathbf{1}}$ \\
\hline No. (\%) of patients & $358(86.1)$ & $58(13.9)$ & \\
Age, years, Me (IQR) & $84(78.8-87.3)$ & $82(76-86)$ & 0.22 \\
Age, 75+, $n(\%)$ & $298(83.2)$ & $52(89.7)$ & 0.22 \\
Gender, women, $n(\%)$ & $279(77.9)$ & $43(74.1)$ & 0.52 \\
Barthel index, Me (IQR) & $95(80-100)$ & $42.5(15-70)$ & $<0.001$ \\
IADL, Me (IQR) & $8(5.0-11.0)$ & $0.0(0.0-4.0)$ & $<0.001$ \\
POMA, Me (IQR) & $23(18-28)$ & $8.0(8.0-15.0)$ & $<0.001$ \\
CFS, Me (IQR) & $4.0(4.0-5.0)$ & $6.0(6.0-7.0)$ & $<0.001$ \\
Not able to walk, $n(\%)$ & $18(5.2)$ & $25(47.2)$ & $<0.001$ \\
Severe frailty, $n(\%)$ & $58(16.2)$ & $44(75.9)$ & $<0.001$ \\
Sarcopenia, $n(\%)$ & $143(43.9)$ & $21(87.5)$ & $<0.001$ \\
Urinary incontinence, $n(\%)$ & $146(41.4)$ & $47(81.0)$ & $<0.001$ \\
Dementia, $n(\%)$ & $102(28.5)$ & $31(53.4)$ & $<0.001$ \\
AMTS, Me (IQR) & $8(6.0-9.0)$ & $18(3.0-8.75)$ & $<0.001$ \\
Neuroleptics, $n(\%)$ & $49(14.0)$ & $14(24.1)$ & 0.001 \\
Parkinson's disease, $n(\%)$ & $41(11.5)$ & $15(25.9)$ & 0.008 \\
TIA/stroke, $n(\%)$ & $41(11.5)$ & $26(46.4)$ & 0.003 \\
MNA-SF $<8, n(\%)$ & $46(13.2)$ & $39.3(3.7)$ & $<0.001$ \\
Albumin, g/L, M (SD) & $36.6(3.6)$ & $<0.001$ \\
\hline
\end{tabular}

$1-\chi^{2}$ test for categorical variables; $t$-test or Mann-Whitney test for continuous or interval variables. In all analyses a two-tailed $P$-value of less than 0.05 was regarded as significant. AMTS-Abbreviated Mental Test Score; CC—calf circumference; CFS - 7-point Clinical Frailty Scale; IADL —instrumental activities of daily living; IQR —interquartile range; $\mathrm{M}-$ mean value; Me —-median value; MNA-SF-Mini Nutritional Assessment Short Form; $\mathrm{n}$-number of cases; POMA—Performance Oriented Mobility Assessment; SD—standard deviation; TIA—-transient ischemic attack. 


\section{Discussion}

Our study has confirmed that falls are a common problem among geriatric ward patients. $40 \%$ of patients in the study group reported falls in the previous year. The percentage of pre-hospitalization falls in our study was similar to that observed by Van Ancum et al. in a study conducted on patients of 70 years of age and older admitted to the academic teaching hospital in Amsterdam [4]. The unfavorable profile of health problems in our patients certainly contributed to this outcome. A high prevalence of such recognized risk factors for falls as Parkinson's disease, osteoarthritis, urinary incontinence, peripheral arterial disease and dependency on others in basic and instrumental activities of daily living was observed in our study participants. Characteristics of gait and balance (gait speed, the POMA score or the TUG score) were significantly worse in the fallers' group, who were also frailer according to the CFS scale.

No significant association with being a faller was observed in our study for many other recognized risk factors for falls. Various studies confirmed, for instance, that more advanced age, female gender, multimorbidity, polypharmacy or taking specific types of medications (for instance neuroleptics, diuretics or antihypertensive medications) increased the risk of falls, but it was not observed in our study $[11,12,33,34]$. We did not note any association between falls and orthostatic hypotension although it was also a well-known risk factor of falls [7]. This fact may have resulted from a retrospective assessment of the occurrence of falls. However, results of other published studies on various risk factors of falls are also slightly contradictory. According to a number of authors, for instance, the use of two or more fall risk-increasing drugs, rather than polypharmacy, is an independent risk factor for falls [35].

Several studies suggested that the nutritional status and BMI should be evaluated when assessing the risk for falls in older age. Systematic review and meta-analysis confirmed a $U$-shaped association observed between BMI and the risk for falls, with the nadir between 24.5 and $30 \mathrm{~kg} / \mathrm{m}^{2}$ [6]. We did not observe this association in our study. The group of fallers had significantly lower scores of MNA-SF and lower levels of albumin, suggesting an association between falls and malnutrition in our study group. Nevertheless, it is certainly worth examining the relationship between falls and nutritional status in geriatric patients in a prospective study, as nutritional biomarkers assessed in the retrospective projection of the study may not necessarily be sensitive enough to properly assess their relationship to falls.

Parkinson's disease was the most significant variable independently associated with falls in our research. It corresponded with the results achieved by other authors [36]. The most important factors related to falls in people with Parkinson's disease comprise of failure of the autonomic nervous system combined with orthostatic hypotension and adverse effects of medications used [37]. Osteoarthritis was another significant variable independently associated with falls in our study and it was consistent with other authors' observations [38].

Contrary to our expectations and to the results of a number of studies, [13] our research did not confirm that dynapenia (suggestive for sarcopenia) was a statistically significant variable associated independently with falls experienced over the last year in patients admitted to the geriatric ward. "Fallers" and "non-fallers" did not differ in the prevalence of dynapenia and severe dynapenia (defined as dynapenia combined with gait speed $\leq 0.8 \mathrm{~m} / \mathrm{s}$ and/or TUG time $\geq 20 \mathrm{~s}$ ). Sarcopenia prevalence differs depending on the population assessed and sarcopenia definition applied. The recently published systematic review and meta-analysis confirmed that older sarcopenic individuals had a significantly higher risk of falls compared with non-sarcopenic individuals, independently of study design, population, sex, sarcopenia definition, continent and study quality [13]. However, several researchers indicate that this analysis may not include important studies in which such a relationship could not be confirmed [39], for example, a study by Henwood et al. conducted in nursing home residents [40] and a study by Schaap et al. conducted in older participants of the Longitudinal Aging Study Amsterdam [41]. Moreover, according to a study by Sim et al. assessing the association between sarcopenia diagnosed with four different criteria and falls-related hospitalization risk in the cohort of 
community-dwelling older Australian women, sarcopenia did not increase the relative hazard ratio for falls-related hospitalization before or after adjustment for age [42]. Unfortunately, many of these assessments did not take into account the independent, simultaneous impact of other determinants of falls significant in older patients, although — as mentioned above-they were multifactorial in nature. Due to the observed tendency of not taking history of falls in the previous year in very frail, functionally dependent and bedridden individuals admitted to the department, it is necessary to consider the possibility of the underestimation of dynapenia/sarcopenia influence on the faller status in our study. Furthermore, sarcopenia and malnutrition are phenomena evolving over time, the risk factors of which are often derived from childhood. These include, for example, a lack of physical activity that contributes to a number of chronic diseases and adverse sequelae [43]. The cross-sectional plan of our study, without data on sarcopenia assessed prior to admission, could also mean that we do not find a correlation between low muscle mass and muscle capacity and falls.

Albeit the assessment of the risk of falls is an important element of the comprehensive geriatric assessment and the question on falls in the last 12 months should be asked at the beginning of the screening, as many as $13.9 \%$ of patients hospitalized in the study period or their guardian, were not asked about or were unable to answer this question. This fact could have been caused by the possible assumption that patients with significantly reduced mobility did not experience falls although the results of a number of studies contradicted this presupposition [44]. A similar tendency was observed in case of the active standing test for detection of orthostatic hypotension [20]. Additionally, in a large number of patients, the hand grip strength, gait speed and TUG test were not assessed (as indicated in Table 1), which could also have influenced the results of the study.

The study has got some limitations that ought to be emphasized. First of all, because of the temporal relationship of fall incidence with baseline medical information, we can say that the study identified potential factors associated with falls, but not actual "predictors" of falls, and the word "predictor" should be treated as a mathematical concept used in regression analysis, rather than as a factor determining the occurrence of a phenomenon. Additionally, as it was underlined above, the study was conducted not in a random sample from the general population, but in a convenient sample of patients admitted to the geriatric ward, therefore, the results can be generalized for patients of similar settings only. Prospective population-based studies should be conducted to explore our findings more thoroughly and overcome these limitations. As our study was based on the secondary analysis of data previously collected, some pieces of information were limited, as indicated in tables. Moreover, the nonparticipant group differed significantly from the study group in various important characteristics. This could also have had an influence on the final results.

\section{Conclusions}

In summary, the study revealed that falls were a common problem among geriatric ward patients and affected up to $43.9 \%$ of them. Parkinson's disease and osteoarthritis were the main independent predictors connected with the significantly higher odds for being a faller before admittance to the hospital. Sarcopenia and some other previously confirmed risk factors for falling were not connected with a faller status in geriatric ward patients, although their influence may be underestimated due to the tendency of not taking the history of falls experienced in very frail, functionally dependent and bedridden individuals.

Author Contributions: Z.B.W. conceived the idea and supervised the study. Z.B.W., A.K. and B.K. were involved in data collection. Z.B.W. and L.M. analyzed and interpreted the data, performed statistical analyses, prepared tables. L.M., M.S., A.K., J.B. and Z.B.W. wrote the manuscript. All authors edited and approved the final version of the manuscript.

Funding: This study was supported by the Medical University of Bialystok, Grant Number N/ST/ZB/15/001/3301 (granted to ZBW). The financial sponsor played no role in the design, execution, analysis and interpretation of data.

Conflicts of Interest: The author declares no conflict of interest. 


\section{Abbreviations}

$\begin{array}{ll}\text { ACE-I } & \text { angiotensin converting enzyme inhibitor } \\ \text { AChE-I } & \text { acetylcholine esterase inhibitor } \\ \text { AMTS } & \text { abbreviated mental test score } \\ \text { ARB } & \text { angiotensin receptor blocker } \\ \text { BDA } & \text { benzodiazepines } \\ \text { BMI } & \text { body mass index } \\ \text { CFS } & \text { Clinical Frailty Scale } \\ \text { CKD-EPI } & \text { Chronic Kidney Disease Epidemiology Collaboration } \\ \text { CI } & \text { confidence interval } \\ \text { GDS } & \text { Geriatric Depression Scale } \\ \text { GFR } & \text { glomerular filtration rate } \\ \text { IADL } & \text { instrumental activities of daily living } \\ \text { IQR } & \text { interquartile range } \\ \text { KDOQI } & \text { Kidney Disease Outcome Quality Initiative } \\ \text { M } & \text { mean } \\ \text { Me } & \text { median } \\ \text { MNA-SF } & \text { Mini Nutritional Assessment Short Form } \\ \text { NSAIDs } & \text { non-steroidal anti-inflammatory drugs } \\ \text { NYHA } & \text { New York Heart Association } \\ \text { OARS } & \text { Older Americans Resources and Services } \\ \text { OR } & \text { odds ratio } \\ \text { POMA } & \text { Performance Oriented Mobility Assessment } \\ \text { SD } & \text { standard deviation } \\ \text { SSRI } & \text { selective serotonin reuptake inhibitors } \\ \text { TIA } & \text { transient ischemic attack } \\ \text { TUG } & \text { Timed Up-and-Go Test } \\ & \end{array}$

\section{References}

1. Tabue-Teguo, M.; Grasset, L.; Avila-Funes, J.A.; Genuer, R.; Proust-Lima, C.; Peres, K.; Féart, C.; Amieva, H.; Harmand, G.-C.; Helmer, C.; et al. Prevalence and Co-Occurrence of Geriatric Syndromes in People Aged 75 Years and Older in France: Results From the Bordeaux Three-city Study. J. Gerontol. A Biol. Sci. Med. Sci. 2017, 73, 109-116. [CrossRef] [PubMed]

2. Wojszel, Z.B.; Bien, B. Falls amongst older people living in the community. Rocz. Akad. Med. Bialymst. 2004, 49, 280-284. [PubMed]

3. Pandya, C.; Magnuson, A.; Dale, W.; Lowenstein, L.; Fung, C.; Mohile, S.G. Association of falls with health-related quality of life (HRQOL) in older cancer survivors: A population based study. J. Geriatr. Oncol. 2016, 7, 201-210. [CrossRef]

4. Van Ancum, J.M.; Pijnappels, M.; Jonkman, N.H.; Scheerman, K.; Verlaan, S.; Meskers, C.G.M.; Maier, A.B. Muscle mass and muscle strength are associated with pre- and post-hospitalization falls in older male inpatients: A longitudinal cohort study. BMC Geriatr. 2018, 18, 116. [CrossRef] [PubMed]

5. Bauer, C.M.; Groger, I.; Rupprecht, R.; Marcar, V.L.; Gassmann, K.G. Prediction of future falls in a community dwelling older adult population using instrumented balance and gait analysis. Z. Gerontol. Geriatr. 2016, 49, 232-236. [CrossRef] [PubMed]

6. Trevisan, C.; Crippa, A.; Ek, S.; Welmer, A.K.; Sergi, G.; Maggi, S.; Manzato, E.; Bea, J.W.; Cauley, J.A.; Decullier, E.; et al. Nutritional Status, Body Mass Index, and the Risk of Falls in Community-Dwelling Older Adults: A Systematic Review and Meta-Analysis. J. Am. Med. Dir. Assoc. 2019, 20, 569-582 e7. [CrossRef]

7. Hartog, L.C.; Schrijnders, D.; Landman, G.W.D.; Groenier, K.; Kleefstra, N.; Bilo, H.J.G.; van Hateren, K.J.J. Is orthostatic hypotension related to falling? A meta-analysis of individual patient data of prospective observational studies. Age Ageing 2017, 46, 568-575. [CrossRef] 
8. Parashos, S.A.; Bloem, B.R.; Browner, N.M.; Giladi, N.; Gurevich, T.; Hausdorff, J.M.; He, Y.; Lyons, K.E.; Mari, Z.; Morgan, J.C.; et al. What predicts falls in Parkinson disease?: Observations from the Parkinson's Foundation registry. Neurol. Clin. Pract. 2018, 8, 214-222. [CrossRef]

9. Ikutomo, H.; Nagai, K.; Tagomori, K.; Miura, N.; Nakagawa, N.; Masuhara, K. Incidence and Risk Factors for Falls in Women With End-Stage Hip Osteoarthritis. J. Geriatr. Phys. Ther. 2019, 42, 161-166. [CrossRef]

10. Tanaka, T.; Matsumoto, H.; Son, B.K.; Imaeda, S.; Uchiyama, E.; Taniguchi, S.; Nishino, A.; Miura, T.; Tanaka, T.; Otsuki, T.; et al. Environmental and physical factors predisposing middle-aged and older Japanese adults to falls and fall-related fractures in the home. Geriatr. Gerontol. Int. 2018, 18, 1372-1377. [CrossRef]

11. Sano, T.; Harada, M.; Sugawara, T.; Isaka, N.; Masuoka, A.; Mikami, A.; Shimamori, Y.; Kurosawa, N. Use of psychotropics and the risk of falls in hospitalized psychiatric patients. Yakugaku Zasshi 2013, 133, 897-903. [CrossRef] [PubMed]

12. Tinetti, M.E.; Han, L.; Lee, D.S.; McAvay, G.J.; Peduzzi, P.; Gross, C.P.; Zhou, B.; Lin, H. Antihypertensive medications and serious fall injuries in a nationally representative sample of older adults. JAMA Intern. Med. 2014, 174, 588-595. [CrossRef] [PubMed]

13. Yeung, S.S.Y.; Reijnierse, E.M.; Pham, V.K.; Trappenburg, M.C.; Lim, W.K.; Meskers, C.G.M.; Maier, A.B. Sarcopenia and its association with falls and fractures in older adults: A systematic review and meta-analysis. J. Cachexia Sarcopenia Muscle 2019, 10, 485-500. [CrossRef]

14. Inouye, S.K.; Studenski, S.; Tinetti, M.E.; Kuchel, G.A. Geriatric syndromes: Clinical, research, and policy implications of a core geriatric concept. J. Am. Geriatr. Soc. 2007, 55, 780-791. [CrossRef] [PubMed]

15. Mazur, K.; Wilczyński, K.; Szewieczek, J. Geriatric falls in the context of a hospital fall prevention program: Delirium, low body mass index, and other risk factors. Clin. Interv. Aging 2016, 11, 1253-1261. [PubMed]

16. Milisen, K.; Coussement, J.; Arnout, H.; Vanlerberghe, V.; De Paepe, L.; Schoevaerdts, D.; Lambert, M.; Van Den Noortgate, N.; Delbaere, K.; Boonen, S.; et al. Feasibility of implementing a practice guideline for fall prevention on geriatric wards: A multicentre study. Int. J. Nurs. Stud. 2013, 50, 495-507. [CrossRef]

17. Cameron, I.D.; Gillespie, L.D.; Robertson, M.C.; Murray, G.R.; Hill, K.D.; Cumming, R.G.; Kerse, N. Interventions for preventing falls in older people in care facilities and hospitals. Cochrane Database Syst. Rev. 2012, 12, Cd005465. [CrossRef]

18. Aizenberg, D.; Weizman, A.; Weiss, A.; Akopian, M.; Amitai, M.; Beloosesky, Y. The Association in Elderly Hospitalized Patients, Between Psychotropic Drugs and Hip Fractures Resulting from Falls. Exp. Aging Res. 2015, 41, 546-555. [CrossRef]

19. Avelino-Silva, T.J.; Farfel, J.M.; Curiati, J.A.E.; Amaral, J.R.G.; Campora, F.; Jacob-Filho, W. Comprehensive geriatric assessment predicts mortality and adverse outcomes in hospitalized older adults. BMC Geriatr. 2014, 14, 129. [CrossRef]

20. Wojszel, Z.B.; Kasiukiewicz, A.; Magnuszewski, L. Health and Functional Determinants of Orthostatic Hypotension in Geriatric Ward Patients: A Retrospective Cross Sectional Cohort Study. J. Nutr. Health Aging 2019, 23, 509-517. [CrossRef]

21. Tinetti, M.E. Performance-oriented assessment of mobility problems in elderly patients. J. Am. Geriatr. Soc. 1986, 34, 119-126. [CrossRef] [PubMed]

22. Podsiadlo, D.; Richardson, S. The timed "Up \& Go": A test of basic functional mobility for frail elderly persons. J. Am. Geriatr. Soc. 1991, 39, 142-148. [PubMed]

23. Roberts, H.C.; Denison, H.J.; Martin, H.J.; Patel, H.P.; Syddall, H.; Cooper, C.; Sayer, A.A. A review of the measurement of grip strength in clinical and epidemiological studies: Towards a standardised approach. Age Ageing 2011, 40, 423-429. [CrossRef] [PubMed]

24. Mahoney, F.I.; Barthel, D.W. Functional Evaluation: The Barthel Index. Md. State Med. J. 1965, 14, 61-65.

25. Fillenbaum, G.G.; Smyer, M.A. The development, validity, and reliability of the OARS multidimensional functional assessment questionnaire. J. Gerontol. 1981, 36, 428-434. [CrossRef]

26. Hodkinson, H.M. Evaluation of a mental test score for assessment of mental impairment in the elderly. 1972. Age Ageing. 2012, 41 (Suppl. 3), iii35-iii40. [CrossRef] [PubMed]

27. Yesavage, J.A. Geriatric Depression Scale. Psychopharmacol. Bull. 1988, 24, 709-711.

28. Rockwood, K.; Song, X.; MacKnight, C.; Bergman, H.; Hogan, D.B.; McDowell, I.; Mitnitski, A. A global clinical measure of fitness and frailty in elderly people. CMAJ 2005, 173, 489-495. [CrossRef] 
29. Kaiser, M.J.; Bauer, J.M.; Ramsch, C.; Uter, W.; Guigoz, Y.; Cederholm, T.; Thomas, D.R.; Anthony, P.; Charlton, K.E.; Maggio, M.; et al. Validation of the Mini Nutritional Assessment short-form (MNA-SF): A practical tool for identification of nutritional status. J. Nutr. Health Aging 2009, 13, 782-788. [CrossRef]

30. Kaufmann, H. Consensus statement on the definition of orthostatic hypotension, pure autonomic failure, and multiple system atrophy. J. Neurol. Sci. 1996, 144, 218-219.

31. Levey, A.S.; Stevens, L.A.; Schmid, C.H.; Zhang, Y.L.; Castro, A.F., 3rd; Feldman, H.I.; Kusek, J.W.; Eggers, P.; Van Lente, F.; Greene, T.; et al. A new equation to estimate glomerular filtration rate. Ann. Intern. Med. 2009, 150, 604-612. [CrossRef]

32. Cruz-Jentoft, A.J.; Bahat, G.; Bauer, J.; Boirie, Y.; Bruyere, O.; Cederholm, T.; Cooper, C.; Landi, F.; Rolland, Y.; Sayer, A.A.; et al. Sarcopenia: Revised European consensus on definition and diagnosis. Age Ageing 2019, 48, 16-31. [CrossRef] [PubMed]

33. Okada, K.; Okada, M.; Kamada, N.; Yamaguchi, Y.; Kakehashi, M.; Sasaki, H.; Katoh, S.; Morita, K. Reduction of diuretics and analysis of water and muscle volumes to prevent falls and fall-related fractures in older adults. Geriatr. Gerontol. Int. 2017, 17, 262-269. [CrossRef] [PubMed]

34. Sotoudeh, G.R.; Mohammadi, R.; Mosallanezhad, Z.; Viitasara, E.; Soares, J.J.F. The prevalence, circumstances and consequences of unintentional falls among elderly Iranians: A population study. Arch. Gerontol. Geriatr. 2018, 79, 123-130. [CrossRef] [PubMed]

35. Zia, A.; Kamaruzzaman, S.B.; Tan, M.P. The consumption of two or more fall risk-increasing drugs rather than polypharmacy is associated with falls. Geriatr. Gerontol. Int. 2017, 17, 463-470. [CrossRef] [PubMed]

36. Kalilani, L.; Asgharnejad, M.; Palokangas, T.; Durgin, T. Comparing the Incidence of Falls/Fractures in Parkinson's Disease Patients in the US Population. PLoS ONE 2016, 11, e0161689. [CrossRef]

37. Schrag, A.; Choudhury, M.; Kaski, D.; Gallagher, D.A. Why do patients with Parkinson's disease fall? A cross-sectional analysis of possible causes of falls. NPJ Parkinsons Dis. 2015, 1, 15011. [CrossRef]

38. Smith, T.O.; Higson, E.; Pearson, M.; Mansfield, M. Is there an increased risk of falls and fractures in people with early diagnosed hip and knee osteoarthritis? Data from the Osteoarthritis Initiative. Int. J. Rheum. Dis. 2018, 21, 1193-1201. [CrossRef]

39. Zhang, X.M.; Cheng, A.S.K.; Dou, Q.; Zhang, W.; Zeng, Y. Comment on: "Sarcopenia and its association with falls and fractures in older adults: A systematic review and meta-analysis" by Yeung et al. J. Cachexia Sarcopenia Muscle 2020, 11, 330-331. [CrossRef]

40. Henwood, T.; Hassan, B.; Swinton, P.; Senior, H.; Keogh, J. Consequences of sarcopenia among nursing home residents at long-term follow-up. Geriatr. Nurs. 2017, 38, 406-411. [CrossRef]

41. Schaap, L.A.; van Schoor, N.M.; Lips, P.; Visser, M. Associations of Sarcopenia Definitions, and Their Components, With the Incidence of Recurrent Falling and Fractures: The Longitudinal Aging Study Amsterdam. J. Gerontol. A Biol. Sci. Med. Sci. 2017, 73, 1199-1204. [CrossRef] [PubMed]

42. Sim, M.; Prince, R.L.; Scott, D.; Daly, R.M.; Duque, G.; Inderjeeth, C.A.; Zhu, K.; Woodman, R.J.; Hodgson, J.M.; Lewis, J.R. Utility of four sarcopenia criteria for the prediction of falls-related hospitalization in older Australian women. Osteoporos. Int. 2019, 30, 167-176. [CrossRef] [PubMed]

43. Booth, F.W.; Roberts, C.K.; Laye, M.J. Lack of exercise is a major cause of chronic diseases. Compr. Physiol. 2012, 2, 1143-1211.

44. Demangeat, J.L.; Geldreich, M.A.; Kessler, B.; Kohlbecker, C.; Sure, M.C.; Jeanmougin, C. Putting into place devices for prevention of falls at the hospital center at Haguenau. Rech. Soins. Infirm. 2009, 99, $26-42$. [CrossRef]

(C) 2020 by the authors. Licensee MDPI, Basel, Switzerland. This article is an open access article distributed under the terms and conditions of the Creative Commons Attribution (CC BY) license (http://creativecommons.org/licenses/by/4.0/). 